\title{
Flow of Thin Liquid Film on an Inclined Solid Surface
}

Khidr M.S. Khidr

College of Science

University of Kirkuk, Iraq

Received on: 01/02/2007

Accepted on: 16/04/2007

\section{ABSTRACT}

In this paper, we consider flow of thin liquid film on an inclined solid surface with an inclination angle $\alpha$. We use the Navier-Stokes equations to obtain equations that govern such flow. We solve these equations analytically with an appropriate boundary conditions to determine the thickness of the film for different values of the inclined angle $\alpha$.

Keywords: flow, thin liquid film, inclined solid surface, Navier-Stokes equations.

$$
\begin{aligned}
& \text { جريان الغثاء السائل على سطح صلب مائل } \\
& \text { خضر محمد صالح خضر }
\end{aligned}
$$

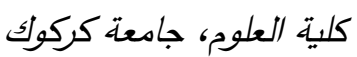

$$
\begin{aligned}
& \text { تاريخ قبول البحث: 2007/04/16 } \\
& \text { تاريخ استلام البحث: 2007/02/01 } \\
& \text { الملخص } \\
& \text { في هذا البحث تم التطرق إلى دراسة الجريان في الأغشية الرقيقة على سطح صلب مائل }
\end{aligned}
$$

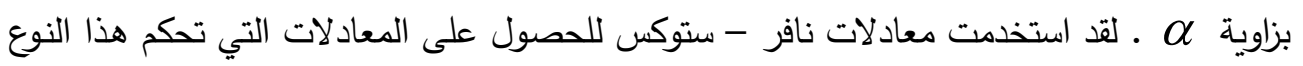

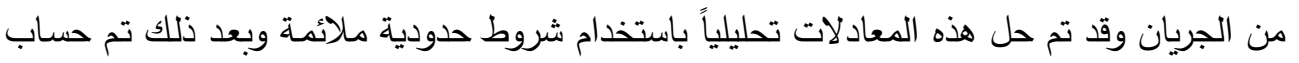

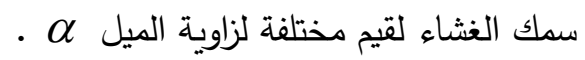

$$
\begin{aligned}
& \text { الكلمات المفتاحية: الجريان، الأغشية الرقيقة، سطح صلب مائل، معادلات نافر - ستوكس. } \\
& \text { المقدمة : Introduction } \\
& \text { إن ميكانيكية الأغشية الرقيقة (Thin films) للسوائل لها أهميـة كبيرة في كثير من }
\end{aligned}
$$

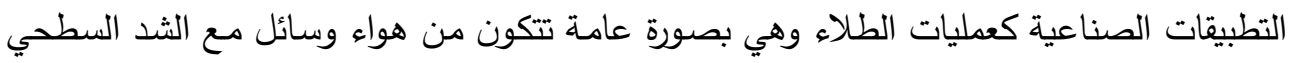

$$
\begin{aligned}
& \text { (2urface tension) } \\
& \text { إذ درس (E.O. Tuck and L. W. Schwartz, 1990) نحافـة }
\end{aligned}
$$

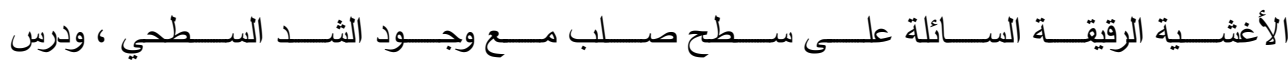

$$
\begin{aligned}
& \text { (Igor. L. Kiakhandler and Gregory I. Sivashinsky, 1996) } \\
& \text { سطح مائل . س س }
\end{aligned}
$$


كما قام كل من (James. J. kriegsmann and Michael J. Miksis, 1998) بدراسة

الأغشية الرقيقة على سطح صلب مائل مـع وجود الثـد السطحي و الجاذبيـة وباستخدام نظريـة التزييت الاعت الترع

ودرس (S. A. Suslov and A. J. Roberts, 1998) خواص الشروط الابتدائية

لنمـوذج التزييت لجريـان الأغثـية الرقيقـة ـ كما درس (Roman O. Grigoriev, 2002)

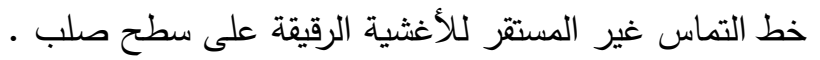

ودرس (D. Gao, N. B. Morley, V. Dhir, 2003) جريـان الأغشية

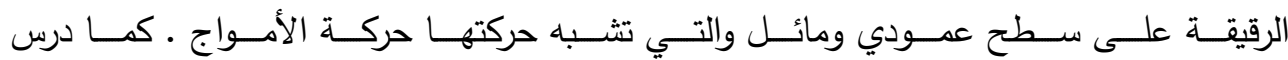

(Lou kondic, Javier A. Diez, 2005) السائلة المائلة عند خط التماس ـ وفي بحثا هذا سوف نتطرق إلى دراسة الجريان اللزج في الأغشية الرقيقة السائلة والمائلة بزاوية م ملى على سطح صلب.

\section{Governing equations : المعادلات التي تحكم الجريان}

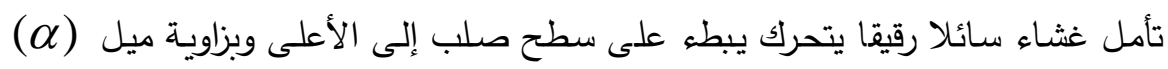

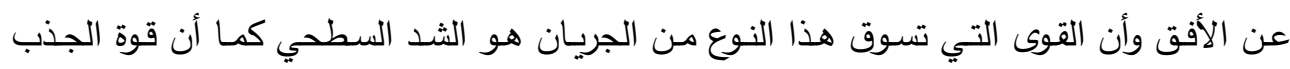

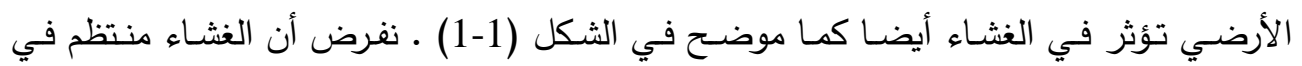
الاتجاه المستعرض (Transvers direction)

لتكن P يمثل الضـغطو u,w دوال لـ (x,z,t) حيث أن السرعة باتجاه ألأحداثي x, x على الترتيب وأن t يمثل الزمن . ليكن معادلة السطح الحر للغشاء بالصيغة آلاتية:

$$
z=h(x, t)
$$

إن معادلات نـافير - ستوكس للجريـان الثنائي البعد باتجاه الاحداثي X,Z على الترتيب

$\rho\left(\frac{\partial u}{\partial t}+u \frac{\partial u}{\partial x}+w \frac{\partial u}{\partial z}\right)=-\frac{\partial p}{\partial x}+\mu\left(\frac{\partial^{2} u}{\partial x^{2}}+\frac{\partial^{2} u}{\partial z^{2}}\right)+\rho g_{x}$

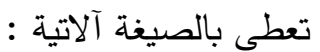
$\rho\left(\frac{\partial w}{\partial t}+u \frac{\partial w}{\partial x}+w \frac{\partial w}{\partial z}\right)=-\frac{\partial p}{\partial z}+\mu\left(\frac{\partial^{2} w}{\partial x^{2}}+\frac{\partial^{2} w}{\partial z^{2}}\right)+\rho g_{z}$

بما أن السائل لزج وان قوى اللزوجة تكون هي المسيطرة على الجريـان يمكن إهمال حد القصور الذاتي وهكذا فأن المعادلتين (2.3) و(2.4) تختزلان إلى الصيغة آلاتية : 


$$
\begin{aligned}
& \frac{\partial p}{\partial x}=\mu \frac{\partial^{2} u}{\partial z^{2}}-\rho g \sin (\alpha) \\
& \frac{\partial p}{\partial z}=-\rho g \cos (\alpha)
\end{aligned}
$$

كما أن معادلة الاستمرارية للجريان الثنائي البعد لها الصيغة آلاتية :

$$
\frac{\partial u}{\partial x}+\frac{\partial w}{\partial z}=0
$$

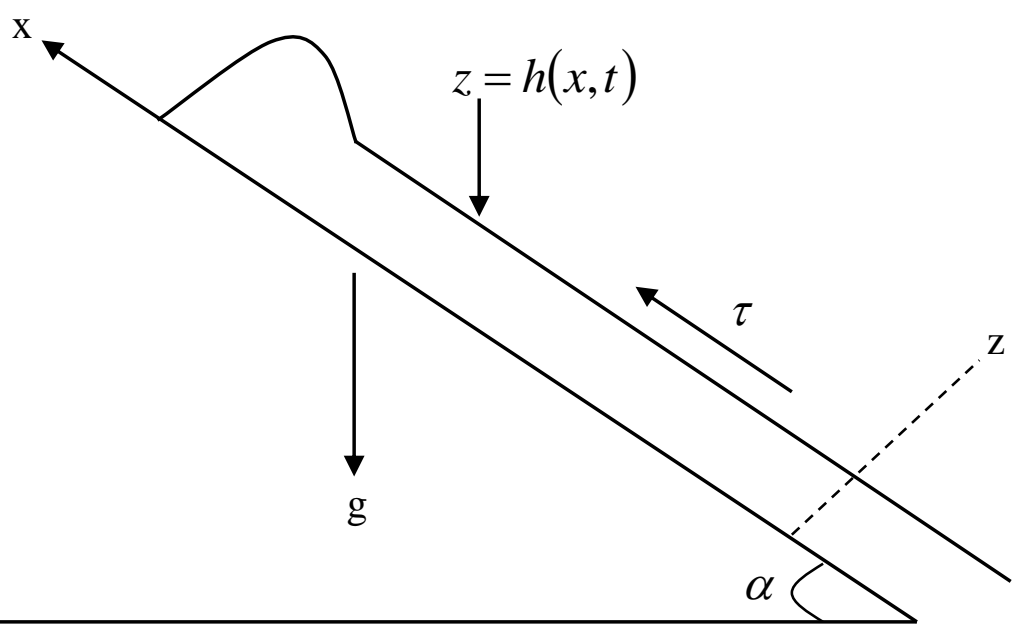

(1.1) الثكل

مقطع عرضي لغثاء رقيق على سطح صلب مائل بزاوية

Boundary conditions : الثروط الحدودية 1. شرط جهد القص : (Tangential stress conditions) على السطح الحر للغشاء

$$
\begin{aligned}
& \tau=\mu\left(\frac{\partial u}{\partial z}\right)_{s}=0 \\
& \text { فأن } \\
& z=h \quad \text { عندما } \\
& \text { حيث أن : } 5 \text { تمثل القيم على السطح الحر للغشاء . } \\
& \text { 2. شرط الجهد العمودي : Normal-stress condition } \\
& p=-\sigma \frac{\partial^{2} h}{\partial x^{2}} \quad \text { عندما } \quad z=h \quad \\
& \text { حيث أن م يمثل الثد السطحي وأن 1>> }
\end{aligned}
$$


3o-slip condition : شرط عدم الانزلاق

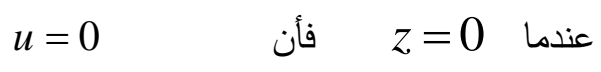

$$
\text { 4. شرط المشتقة التي تتبع الجسيم على السطح الحر . }
$$

فالمشتقة المادية (المشتقة التابعة) Material derivative هي المشتقة التي تتبع الجسيم على لئى $\frac{D}{D t}=\frac{\partial}{\partial t}+u \frac{\partial}{\partial x}+w \frac{\partial}{\partial z}=0$ السطح الحر وتعرف رياضياً بالصيغة : $F(x, z, t)=z-h(x, t)$ يمكن كتابة معادلة السطح الحر للغشاء (2.2) بالصيغة : بما أن السطح الحر يمثل خط انسياب فأن المشتقة المادية تساوي صفرا أي أن : $\frac{D F}{D t}=\frac{\partial F}{\partial t}+u \frac{\partial F}{\partial x}+w \frac{\partial F}{\partial z}=0$

$-\frac{\partial h}{\partial t}-u \frac{\partial h}{\partial x}+w=0$ $z=h$ عندما وبتكامل المعادلة (2.7) بالنسبة الى z وتعويضها في المعادلة (3.5) عندما $-\frac{\partial h}{\partial t}-u \frac{\partial h}{\partial x}-h \frac{\partial u}{\partial x}=0$ $-\frac{\partial h}{\partial t}-\frac{\partial}{\partial x}(u h)=0$

$-\frac{\partial h}{\partial t}=\frac{\partial}{\partial x}(u h)$

(4.1) الأشتقاقات والتكاملات اللازمة لإيجاد المعادلة التي تحكم الجريان :

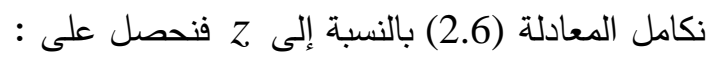
$p(x, t)=-\rho g \cos (\alpha) z+f_{1}(x, t)$

$$
\text { حيث أن : }
$$
بمقارنة المعادلة (4.2) مع الشرط الحدي (3.3) عندما $-\sigma \frac{\partial^{2} h}{\partial x^{2}}=-\rho g \cos (\alpha) h+f_{1}(x, t)$ 
$f_{1}(x, t)=-\sigma \frac{\partial^{2} h}{\partial x^{2}}+\rho g \cos (\alpha) h$

نعوض المعادلة (4.3) في المعادلة (4.2) فنحصل على :

$p(x, t)=-\rho g \cos (\alpha) z-\sigma \frac{\partial^{2} h}{\partial x^{2}}+\rho g \cos (\alpha) h$

نثتق المعادلة (4.4) بالنسبة إلى $x$ فنحصل على :

$\frac{\partial p}{\partial x}=-\sigma \frac{\partial^{3} h}{\partial x^{3}}+\rho g \cos (\alpha) \frac{\partial h}{\partial x}$

نقارن المعادلة (4.5) مع المعادلة (2.5) فنحصل على :

$\mu \frac{\partial^{2} u}{\partial z^{2}}-\rho g \sin (\alpha)=-\sigma \frac{\partial^{3} h}{\partial x^{3}}+\rho g \cos (\alpha) \frac{\partial h}{\partial x}$

نكامل المعادلة (4.6) بالنسبة إلى z فنحصل على :

$\mu \frac{\partial u}{\partial z}=\rho g \sin (\alpha) z-\sigma \frac{\partial^{3} h}{\partial x^{3}} z+\rho g \cos (\alpha) \frac{\partial h}{\partial x} z+f_{2}(x, t)$

حيث أن $f_{2}$ ثابت التكامل .

باستخدام الشرط الحدي (3.2) عندما

$0=\rho g \sin (\alpha) h-\sigma \frac{\partial^{3} h}{\partial x^{3}} h+\rho g \cos (\alpha) \frac{\partial h}{\partial x} h+f_{2}(x, t)$

$f_{2}(x, t)=-\rho g \sin (\alpha) h+\sigma \frac{\partial^{3} h}{\partial x^{3}} h-\rho g \cos (\alpha) \frac{\partial h}{\partial x} h$

: في المعادلة (4.7) فنحصل على :

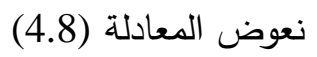

$\mu \frac{\partial u}{\partial z}=\rho g \sin (\alpha) z-\sigma \frac{\partial^{3} h}{\partial x^{3}} z+\rho g \cos (\alpha) \frac{\partial h}{\partial x} z-$

$$
\rho g \sin (\alpha) h+\sigma \frac{\partial^{3} h}{\partial x^{3}} h-\rho g \cos (\alpha) \frac{\partial h}{\partial x} h
$$

نكامل المعادلة (4.9) بالنسبة إلى z فنحصل على :

$\mu u=\rho g \sin (\alpha) \frac{z^{2}}{2}-\sigma \frac{\partial^{3} h}{\partial x^{3}} \frac{z^{2}}{2}+\rho g \cos (\alpha) \frac{\partial h}{\partial x} \frac{z^{2}}{2}-\rho g \sin (\alpha) h z+$

$$
\begin{aligned}
& \sigma \frac{\partial^{3} h}{\partial x^{3}} h z-\rho g \cos (\alpha) \frac{\partial h}{\partial x} h z+f_{3}(x, t) \\
& \text { حيث أن } f_{3} \text { ثابت التكامل . } \\
& \text { باستخدام الثرط الحدي (3.4) عندما } 0=0 \\
& f_{3}(x, t)=0
\end{aligned}
$$




$$
\text { نعوض المعادلة (4.11) في المعادلة (4.10) عندما }
$$

$\mu u=-\frac{\rho g}{2} \sin (\alpha) h^{2}-\frac{\rho g}{2} \cos (\alpha) \frac{\partial h}{\partial x} h^{2}+\frac{\sigma}{2} \frac{\partial^{3} h}{\partial x^{3}} h^{2}$

$$
\text { بقسمة المعادلة (4.12) على }
$$

$u h=-\frac{\rho g}{2 \mu} \sin (\alpha) h^{3}-\frac{\rho g}{2 \mu} \cos (\alpha) \frac{\partial h}{\partial x} h^{3}+\frac{\sigma}{2 \mu} \frac{\partial^{3} h}{\partial x^{3}} h^{3}$

نشتق المعادلة (4.13) بالنسبة الى $x$ نحصل على :

$\frac{\partial}{\partial x}(u h)=\frac{\partial}{\partial x}\left\{-\frac{\rho g}{2 \mu} \sin (\alpha) h^{3}-\frac{\rho g}{2 \mu} \cos (\alpha) \frac{\partial h}{\partial x} h^{3}+\frac{\sigma}{2 \mu} \frac{\partial^{3} h}{\partial x^{3}} h^{3}\right\}$

بتعويض المعادلة (3.6) في المعادلة (4.14) نحصل على :

$-\frac{\partial h}{\partial t}=\frac{\partial}{\partial x}\left\{-\frac{\rho g}{2 \mu} \sin (\alpha) h^{3}-\frac{\rho g}{2 \mu} \cos (\alpha) \frac{\partial h}{\partial x} h^{3}+\frac{\sigma}{2 \mu} \frac{\partial^{3} h}{\partial x^{3}} h^{3}\right\}$

Steady state : الحالة اللازمنية

في حالة الجريان اللازمني فأن المعادلة (4.15) تصبح بالصيغة آلاتية :

$$
\begin{aligned}
& \frac{\partial}{\partial x}\left\{-\frac{\rho g}{2 \mu} \sin (\alpha) h^{3}-\frac{\rho g}{2 \mu} \cos (\alpha) \frac{\partial h}{\partial x} h^{3}+\frac{\sigma}{2 \mu} \frac{\partial^{3} h}{\partial x^{3}} h^{3}\right\}=0 \\
& -\frac{\rho g}{2 \mu} \frac{\partial}{\partial x} \sin (\alpha) h^{3}-\frac{\rho g}{2 \mu} \frac{\partial}{\partial x} \cos (\alpha) \frac{\partial h}{\partial x} h^{3}+\frac{\sigma}{2 \mu} \frac{\partial}{\partial x} \frac{\partial^{3} h}{\partial x^{3}} h^{3}=0 \\
& \text { نضرب المعادلة (5.3) في } \\
& \frac{\partial}{\partial x}\left\{\sin (\alpha) h^{3}+\cos (\alpha) \frac{\partial h}{\partial x} h^{3}-\frac{\sigma}{\rho g} \frac{\partial^{3} h}{\partial x^{3}} h^{3}\right\}=0
\end{aligned}
$$

Non-dimensional variables: المتغيرات اللابعدية

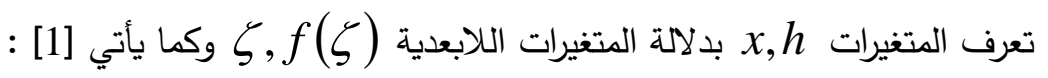




$$
\left.\begin{array}{l}
x=\left(\frac{\sigma}{\rho g}\right)^{\frac{1}{2}} \zeta \\
h=\left(\frac{\sigma}{\rho g}\right)^{\frac{1}{2}} f(\zeta)
\end{array}\right\}
$$

نعوض المعادلة (6.2) في المعادلة (5.4) فنحصل على :

$\frac{\partial}{\partial x}\left\{\sin (\alpha)\left(\frac{\sigma}{\rho g}\right)^{\frac{3}{2}} f^{3}+\cos (\alpha)\left(\frac{\sigma}{\rho g}\right)^{\frac{3}{2}} f^{3} f^{\prime}-\left(\frac{\sigma}{\rho g}\right)^{\frac{3}{2}} f^{3} f^{\prime \prime \prime}\right\}=0$

$$
\text { بضرب المعادلة (6.3) في }
$$

$$
\frac{\partial}{\partial x}\left\{\sin (\alpha) f^{3}+\cos (\alpha) f^{3} f^{\prime}-f^{3} f^{\prime \prime \prime}\right\}=0
$$

نكامل المعادلة (6.4) فنحصل على :

$$
\sin (\alpha) f^{3}+\cos (\alpha) f^{3} f^{\prime}-f^{3} f^{\prime \prime \prime}=H
$$

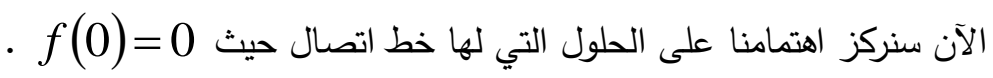

وان خط الاتصال (خط التماس) Contact line هو خط أو نقطة التقاء السائل مع سطح

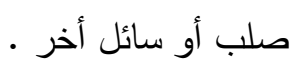

نفترض أن خط الاتصال يقع عند 0 = ك كذلك عندما م-

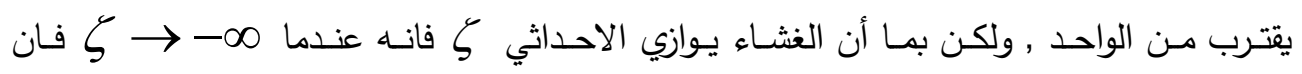

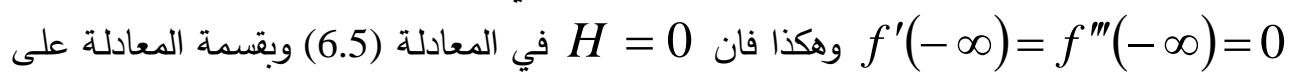
f $f^{3}$

$$
f^{\prime \prime \prime}-\cos (\alpha) f^{\prime}=\sin (\alpha)
$$

نكامل المعادلة (6.6) فنحصل على :

$$
f^{\prime \prime}-\cos (\alpha) f=\sin (\alpha) \zeta+A_{1}
$$

وان الحل العام للمعادلة (6.7) هو :

$$
\begin{aligned}
& f(\zeta)=c_{1} e^{\sqrt{\cos (\alpha)} \zeta}+c_{2} e^{-\sqrt{\cos (\alpha)} \zeta}-\tan (\alpha) \zeta-\frac{A_{1}}{\cos (\alpha)} \\
& \zeta=0 \Rightarrow f=0
\end{aligned}
$$




$$
\zeta=-5 \Rightarrow f=1
$$

نعوض الشروط الابتدائية في الحال العام فنحصل على :

$c_{2}=\frac{1+\tan (\alpha)+\frac{A_{1}}{\cos (\alpha)}-\frac{A_{1}}{\cos (\alpha)} e^{\sqrt{\cos (\alpha)}}}{e^{-\sqrt{\cos (\alpha)}}-e^{\sqrt{\cos (\alpha)}}}$

$c_{1}=\frac{A_{1}}{\cos (\alpha)}-c_{2}$

بتعويض الثوابت

$f(\zeta)=\left(\frac{A_{1}}{\cos (\alpha)}-c_{2}\right) e^{\sqrt{\cos (\alpha)} \zeta}+c_{2} e^{-\sqrt{\cos (\alpha)} \zeta}-\tan (\alpha) \zeta-\frac{A_{1}}{\cos (\alpha)}$

إن حلول المعادلة (6.7) موضحة في الثكل (1.2) عندما 1 (a 1 (a)

والثكل (1.3) يوضح عندما

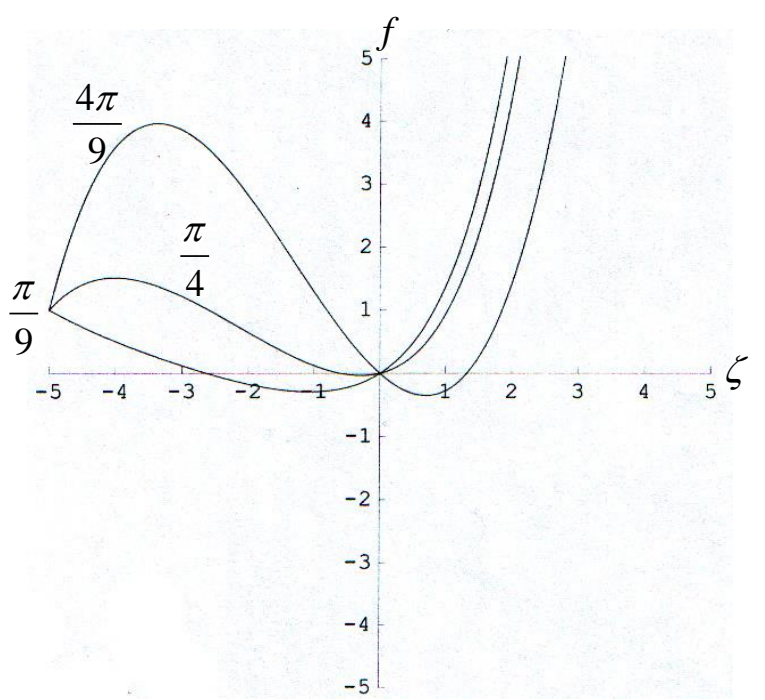

(1.2) (الثكل) (1.2)

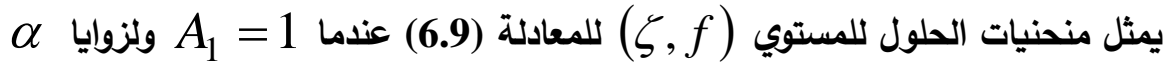




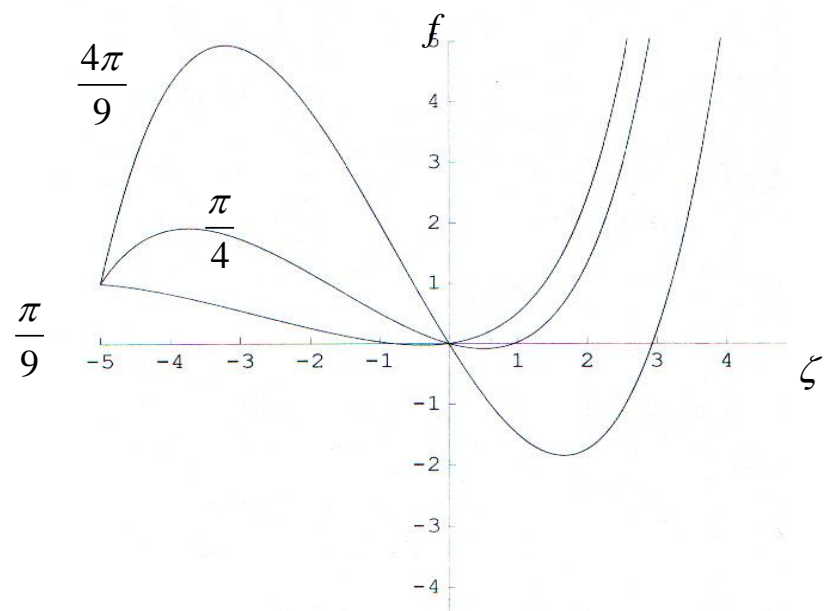

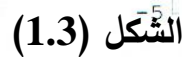

يمثل منحنيات الحلول للمستوي (f,f) للمعادلة (6.9) عندما

Conclusions : الاستنتاجات

في دراستتا للجريـان للازمني للأغشية الرقيقة السائلة على سطح صلب مائل تبين من

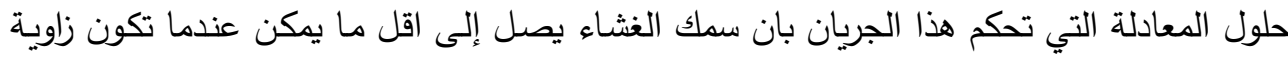

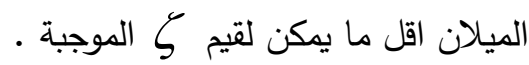
إما قيم ك السالبة فان سمك الغشاء بدأ بالتذبذب إلى إن يصل قيمته المحاذية أي انه

$$
\text { عندما }
$$




$$
\begin{aligned}
& \text { المصادر }
\end{aligned}
$$

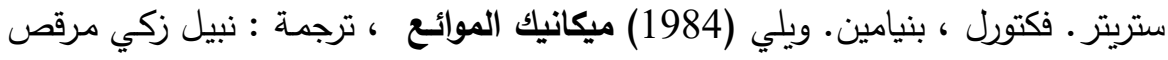

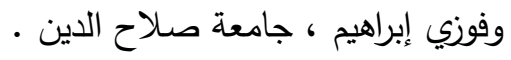

$$
\begin{aligned}
& \text { سعيد ، مهند عاصم (2002) الجريان اللازمني في الاغشية الرقيقة بإهمال قوى ، إنى } \\
& \text { القصور الذاتي ، أطروحة ماجستير ، كلية التربية ، جامعة الموصل } 2002 \text {. }
\end{aligned}
$$

[3] Cao, D.,N.B.NMorley, V.Dhin (2003) "Numerical simulation of wavy falling film flow using VOF method" Journal of computational physics 192624-642.

[4] Igor,L.Kliakhandler and Gregory I. Sivashinsky (1996) "Viscous damping and instabilities in stratified liquid film flowing down a slightly inclined plane" Phys.Fluids, Vol.9 No.1,January .

[5] James, J.Kriegsmann and Michael J. Miksis (1998) "Pressure driven disturbances on a thin viscous film" Phys.Fluids, Vol.10,No.6,June .

[6] Lou Kondic,Javier A.Dies "On nontrivial traveling waves in thin film flows including contact lines" Physics D 209(2005)135-144.

[7] Raman, O.Grigoriev (2002) "Contact line Instability and pattern Selection in thermally driven liquid films" November.

[8] Lou Kondic,Javier A.Dies "On nontrivial traveling waves in thin film flows including contact lines" Physics D 209(2005)135-144.

[8] Suslov, S.A. and A.J.Roberts (1998) "Proper initial conditions for the lubrication model of the flow of a thin film of fluid" ar Xivichao -dyn/9804018 Vol.8 Apr.

[9] Tuck,E.O. and L.W.Schwartz (1990)"Numerical and asymptotic study of some third-order ordinary differential equations relevant to draining and coating flows",No.3,pp.453-469,September . 